\title{
Neurons in the Intermediate Reticular Nucleus Coordinate Postinspiratory Activity, Swallowing, and Respiratory- Sympathetic Coupling in the Rat
}

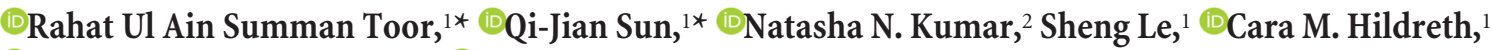 \\ ㄱocqueline K. Phillips, ${ }^{1 \star}$ and ${ }^{\oplus S}$ Simon McMullan ${ }^{1 \star}$ \\ ${ }^{1}$ Department of Biomedical Sciences, Faculty of Medicine and Health Sciences, Macquarie University, 2109 New South Wales, Australia, and ${ }^{2}$ Department \\ of Pharmacology, School of Medical Science, University of New South Wales, 2052 New South Wales, Australia
}

Breathing results from sequential recruitment of muscles in the expiratory, inspiratory, and postinspiratory (post-I) phases of the respiratory cycle. Here we investigate whether neurons in the medullary intermediate reticular nucleus (IRt) are components of a central pattern generator (CPG) that generates post-I activity in laryngeal adductors and vasomotor sympathetic nerves and interacts with other members of the central respiratory network to terminate inspiration. We first identified the region of the (male) rat IRt that contains the highest density of lightly cholinergic neurons, many of which are glutamatergic, which aligns well with the putative postinspiratory complex in the mouse (Anderson et al., 2016). Acute bilateral inhibition of this region reduced the amplitudes of post-I vagal and sympathetic nerve activities. However, although associated with reduced expiratory duration and increased respiratory frequency, IRt inhibition did not affect inspiratory duration or abolish the recruitment of post-I activity during acute hypoxemia as predicted. Rather than representing an independent CPG for post-I activity, we hypothesized that IRt neurons may instead function as a relay that distributes post-I activity generated elsewhere, and wondered whether they could be a site of integration for para-respiratory CPGs that drive the same outputs. Consistent with this idea, IRt inhibition blocked rhythmic motor and autonomic components of fictive swallow but not swallow-related apnea. Our data support a role for IRt neurons in the transmission of post-I and swallowing activity to motor and sympathetic outputs, but suggest that other mechanisms also contribute to the generation of post-I activity.

Key words: brainstem; pons; postinspiration; respiration; respiratory-sympathetic coupling; sympathetic

Significance Statement

Interactions between multiple coupled oscillators underlie a three-part respiratory cycle composed from inspiratory, postinspiratory (post-I), and late-expiratory phases. Central post-I activity terminates inspiration and activates laryngeal motoneurons. We investigate whether neurons in the intermediate reticular nucleus (IRt) form the central pattern generator (CPG) responsible for post-I activity. We confirm that IRt activity contributes to post-I motor and autonomic outputs, and find that IRt neurons are necessary for activation of the same outputs during swallow, but that they are not required for termination of inspiration or recruitment of post-I activity during hypoxemia. We conclude that this population may not represent a distinct CPG, but instead may function as a premotor relay that integrates activity generated by diverse respiratory and nonrespiratory CPGs.

\section{Introduction}

Breathing is a fundamental motor function controlled by groups of spatially, functionally, and neurochemically compartmental-

\footnotetext{
Received March 4, 2019; revised 0ct. 4, 2019; accepted 0ct. 15, 2019.

Author contributions: R.U.A.S.T., Q.-J.S., N.N.K., C.M.H., J.K.P., and S.M. designed research; R.U.A.S.T., Q.-J.S., N.N.K., S.L., and S.M. performed research; R.U.A.S.T., Q.-J.S., and S.M. analyzed data; R.U.A.S.T. wrote the first draft of the paper; R.U.A.S.T., Q.-J.S., N.N.K., C.M.H., J.K.P., and S.M. edited the paper; S.M. wrote the paper.

This work was supported by the Australian Research Council Discover Project DP120100920, National Health and Medical Research Council APP1156727, and the Hillcrest Foundation. The postgraduate studies of R.U.A.S.T. and S.L. were supported by Macquarie University research scholarships.

S.L. is an employee of Olympus Australia. The remaining authors declare no competing financial interests.
}

ized neurons in the brainstem and pons (for review, see Alheid and McCrimmon, 2008; Feldman et al., 2013; Smith et al., 2013; Del Negro et al., 2018). In quiet breathing (eupnea), respiratory muscles are recruited in three distinct phases of activity: lateexpiration, inspiration, and postinspiration. The functions of the expiratory and inspiratory phases are self-explanatory, but the

*R.U.A.S.T., Q.-J.S., J.K.P. and S.M. contributed equally to this work. Correspondence should be addressed to Simon McMullan at simon.mcmullan@mq.edu.au. https://doi.org/10.1523/JNEUROSCI.0502-19.2019

Copyright $\odot 2019$ the authors 
physiological significance of the postinspiratory (post-I) phase is less obvious; it is characterized by laryngeal adduction and contraction of the crural diaphragm, which act together to slow expiratory airflow and may consequently facilitate gas exchange (Dutschmann et al., 2014). Post-I activity also manifests in the activity of sympathetic premotor neurons and is the dominant component of respiratory-sympathetic coupling in rat splanchnic, renal, lumbar, and thoracic sympathetic outputs (Haselton and Guyenet, 1989; Guyenet et al., 1990; Häbler et al., 1994; Miyawaki et al., 1995; Simms et al., 2009; Moraes et al., 2013; Menuet et al., 2017). It too is suggested as a way of optimizing gas exchange by coordinating tissue perfusion with ventilation, and of enhancing the dynamic ranges of both systems (Zoccal et al., 2009; Simms et al., 2010; Dick et al., 2014).

The mechanisms responsible for generating respiratory post-I activity are controversial. One view is that post-I depolarization of laryngeal motoneurons reflects rebound from intense inhibition received during the late expiratory and inspiratory periods, which originates in the Bötzinger Complex (BötC) and preBötzinger Complex (preBötC), respectively. In this model, excitatory drive to laryngeal motoneurons plays a minor role in the generation of post-I activity (Dutschmann and Paton, 2002; Ono et al., 2006; Rybak et al., 2007; Sun et al., 2008; Bautista et al., 2010). However, this mechanism is unlikely to underlie post-I activity in sympathetic premotor neurons in the rostral ventrolateral medulla (RVLM), which results from excitatory synaptic input during the post-I period (Lipski et al., 1996; Moraes et al., 2013) and is abolished by blockade of glutamatergic, but not GABAergic or glycinergic, inputs (Guyenet et al., 1990; Miyawaki et al., 2002). Furthermore, RVLM sympathetic premotor neurons do not receive significant input from BötC neurons (Dempsey et al., 2017; Menuet et al., 2017), a major source of inhibitory input to post-I laryngeal motoneurons (Jiang and Lipski, 1990; Ono et al., 2006). So where does post-I excitatory drive come from?

Anderson et al. (2016) recently challenged the "inhibitory rebound" model with the discovery of a group of excitatory cholinergic interneurons in the mouse intermediate reticular nucleus (IRt), which depolarize in the post-I period in a neonatal slice preparation. In vivo stimulation of cholinergic or glutamatergic neurons in this region, which they named the post-I complex (PiCo), resulted in post-I-like activity in the cervical vagus nerve, whereas inhibition reduced post-I activity. Based on these observations, they suggested that this cell group is the neural correlate of post-I activity, and that its behavior is necessary and sufficient for the generation of the post-I phase.

Here we investigated whether activity in the region described by Anderson et al. (2016) also underlies the generation of post-I sympathetic (and respiratory) activities in anesthetized, vagotomized, and artificially ventilated rats. We first established the presence of neurons in the rat IRt that correspond well neuroanatomically and phenotypically with the mouse PiCo. Subsequent loss-of-function experiments suggested that activity in this region contributes to eupneic post-I activity, but not to the enhanced post-I drive seen during acute hypoxemia. Consequently, we tested the hypothesis that the population described by Anderson et al. (2016) may not reflect the post-I central pattern generator (CPG) per se, but instead represents a relay that coordinates drive from multiple respiratory and nonrespiratory CPGs to common motor and autonomic outputs. In support of our hypothesis, we found that, while IRt activity underlies the transmission of rhythmic activity to laryngeal and sympathetic outputs, it does not contribute to the apneic component of the swallow reflex.

\section{Materials and Methods}

Ethics approval. Experiments were performed on adult ChAT-Cre mice of either sex (The Jackson Laboratory, strain 006410) or male Lewis rats (350-450 g; Animal Resource Centre, Perth, Western Australia, Australia), conducted in accordance with the Australian Code for the care and use of animals for scientific purposes and approved by the Macquarie University Animal Ethics Committee.

Anatomy experiments. Animals were killed with $150 \mathrm{mg} / \mathrm{kg}$ sodium pentobarbitone intraperitoneally and immediately perfused transcardially with heparinized saline followed by $4 \%$ formaldehyde. Brains were postfixed overnight and cut into $35 \mu \mathrm{m}$ (mice) or $50 \mu \mathrm{m}$ (rats) coronal sections and every third (mice) or fourth (rats) section processed.

Sections from mice were visualized chromogenically using DAB precipitation. Briefly, sections were blocked with 50\% EtoH (30 min), then $1 \% \mathrm{H}_{2} \mathrm{O}_{2}$ (peroxidase block, $30 \mathrm{~min}$ ). Thereafter they were incubated overnight in primary antibody (goat anti-ChAT, Merck Millipore, AB144P, 1:200), $0.05 \%$ merthiolate (T5125, Sigma-Aldrich) with $10 \%$ normal horse serum (Jackson ImmunoResearch Laboratories), followed after washing by overnight incubation in biotinylated donkey anti-goat antibody (1:500, Jackson ImmunoResearch Laboratories). The next day, sections were incubated for an hour with avidin-biotin complex (Vectastain ABC Kit, PK-6100, Vector Laboratories). Nickel-conjugated DAB solution (SK-4100, Vector Laboratories) was added to develop black cytoplasmic staining. Sections were dehydrated and coverslipped with DPX mounting media.

Sections from rats were processed with the same primary antibody ( $1: 800,48-72 \mathrm{~h})$ but visualized with fluorescent secondary antibodies: following incubation with primary antibodies, sections were washed $3 \times$ $15 \mathrm{~min}$ in TPBS and incubated overnight in secondary antibody (donkey anti-sheep IgG conjugated to AlexaFluor-555, 1:500, Invitrogen, \#A21436/AB_2535857) with $2 \%$ normal horse serum, washed again, and mounted on microscope slides in Vectorshield mounting medium (Vector Laboratories) and imaged using a Z3 epifluorescence microscope (Carl Zeiss) or SP5 confocal (Carl Zeiss).

ChAT-immunoreactive $\left(\mathrm{ChAT}^{+}\right)$IRt neurons from 3 ChAT-cre mice and 3 Lewis rats were mapped using a volumetric atlas as previously described (Dempsey et al., 2017). In brief, montage images of sections lying between the hypoglossal nucleus and the middle of the facial nucleus were captured $(10 \times / 0.30$ NA M27 objective lens), and labeled neurons were manually annotated. Sections from mice or rats were aligned to the Allen or Waxholm (Papp et al., 2014) volumetric brain atlases, respectively, using the QuickNII alignment tool (Bjerke et al., 2018) (https://www.nitrc.org/projects/quicknii), transforming the pixel coordinates of neurons identified in 2D images to 3D Cartesian coordinates. For each mouse, the distribution of $\mathrm{ChAT}^{+}$IRt neurons was projected into $2 \mathrm{D}$ grayscale heat maps using a 5 voxel smoothing coefficient, as described previously (Dempsey et al., 2017). Grayscale heat maps were averaged between replicates before colorization in ImageJ and plotted onto corresponding atlas sections. A 3D density mapping algorithm was used to define the anatomical boundary that included $66 \%$ of the $\mathrm{ChAT}^{+}$IRt neurons in the rat (Burguet and Andrey, 2014; Farmer et al., 2019).

Cholinergic IRt neurons from a separate group of Lewis rats were examined for colocalization of ChAT immunoreactivity with vesicular glutamate transporter 2 (VGluT2) mRNA revealed by ISH. A digoxigeninlabeled cRNA probe for VGluT2 was synthesized from a cDNA template (886 bp, GenBank reference NM_053427) and amplified using PCR primers (lowercase) with T7 and Sp6 RNA polymerase promotors (uppercase): forward, GGATCCATTTAGGTGACACTATAGAAGtcaatgaaatccaacgtcca; reverse, GAATTCTAATACGACTCACTATAGGGAGAcaagagcacaggacac caaa.

Following purification of the DNA template with gel extraction, antisense riboprobes were in vitro transcribed (Epicenter Technologies) incorporating digoxigenin-11-UTP (Roche Applied Science) and validated as previously described (Kumar et al., 2012). Brainstem sections were incubated in prehybridization buffer ( $50 \%$ formamide, $100 \mu \mathrm{g} / \mathrm{ml}$ heparin, $5 \times$ SSC, pH 7.0, $1 \times$ Denhardt's solution, $250 \mu \mathrm{g} / \mathrm{ml}$ herring sperm DNA, $100 \mu \mathrm{g} / \mathrm{ml}$ yeast tRNA, $5 \%$ dextran sulfate, $0.1 \%$ Tween 20 , SigmaAldrich, unless otherwise indicated $)$ at $37^{\circ} \mathrm{C}(30 \mathrm{~min})$, then $58^{\circ} \mathrm{C}(1 \mathrm{~h})$ 
before hybridization with gentle agitation with riboprobe $(1000 \mathrm{ng} / \mathrm{ml})$ at $58^{\circ} \mathrm{C}(12-18 \mathrm{~h})$. Sections were washed in $2 \times$ SSC buffer with $0.1 \%$ Tween 20 , followed by $0.2 \times$ SSC buffer with $0.1 \%$ Tween 20 , and then maleic acid buffer ( $0.1 \mathrm{~m}$ maleic acid, $0.15 \mathrm{M} \mathrm{NaCl}, 0.1 \%$ Tween 20$)$. The tissue was then blocked in maleic acid buffer containing $2 \%$ Boehringer blocking reagent (Roche Applied Science) and 10\% normal horse serum.

Goat anti-ChAT (1:400) was added to the blocking buffer and incubated at $4^{\circ} \mathrm{C}(48 \mathrm{~h})$. Sections were washed in TBSm $(100 \mathrm{~mm}$ Tris-HCl, $150 \mathrm{~mm} \mathrm{NaCl}, \mathrm{pH} 7.4,0.05 \%$ merthiolate, $3 \times 30 \mathrm{~min}$ ) and incubated overnight with AlexaFluor-594 donkey anti-goat IgG secondary antibody (1:400, Jackson ImmunoResearch Laboratories, catalog \#705-585147) with $1 \%$ normal horse serum. DIG-labeled neurons were revealed by incubation in NTMT $(0.1 \mathrm{M} \mathrm{NaCl}, 0.1 \mathrm{~m}$ Tris- $\mathrm{HCl}, \mathrm{pH}$ 9.5, $0.1 \mathrm{M}$ $\mathrm{MgCl}_{2}, 0.1 \%$ Tween 20, 2 mM tetramisole $\mathrm{HCl}$ ) containing NBT (Roche Applied Science) and BCIP salts (Roche Applied Science). The reaction was stopped by washing $(0.1 \mathrm{~m}$ Tris, $1 \mathrm{~mm}$ EDTA, $\mathrm{pH} 8.5,3 \times 15 \mathrm{~min})$ when DIG labeling was intense with minimal background staining. No labeling was seen when the sense probe was substituted for the antisense probe.

The proportion of $\mathrm{ChAT}^{+}$IRt neurons that contained VGluT2 ISH product was estimated from confocal scans that captured immunofluorescence and brightfield channels $(20 \times$ objective, $750 \times 750 \mu \mathrm{m}, 1024 \times$ 1024 pixels), taken with the center of the FOV positioned dorsomedial to nucleus ambiguus at the same rostrocaudal level as the caudal pole of the facial nucleus. $\mathrm{ChAT}^{+} \mathrm{VGluT}^{+}{ }^{+}$neurons were manually annotated in two images from each of three experimental replicates.

Physiology experiments: general surgical preparation. Physiological experiments were performed on Lewis rats initially anesthetized with a bolus of $10 \%$ urethane ( $10 \% \mathrm{w} / \mathrm{v}$ in saline, $1.1-1.3 \mathrm{~g} / \mathrm{kg}$, i.p.) and supplemented with additional doses $(130 \mathrm{mg}$, i.v. $)$ as required. Depth of anesthesia was assessed by checking for absence of withdrawal and/or arterial blood pressure $(>10 \mathrm{mmHg})$ responses to paw pinch. The right femoral artery and vein were cannulated, and tracheostomy was performed to allow for mechanical ventilation with oxygen enriched room air (final oxygen concentration $>50 \%$ ) such that arterial blood $\mathrm{pCO}_{2}$ and $\mathrm{pH}$ were maintained at $45-55 \mathrm{mmHg}$ and $7.35-7.45$, respectively, which were measured by periodic analysis with a VetStat electrolyte and blood gas analyzer (IDEXX). Where indicated, metabolic acidosis was treated with $5 \%$ sodium bicarbonate $(0.2-0.5 \mathrm{ml}$, i.v., Merck). Core temperature was measured with a rectal probe and maintained at $\sim 37^{\circ} \mathrm{C}$ with a homeothermic blanket (Harvard Apparatus). Animals were bilaterally vagotomized to desynchronize central respiratory drive from afferent feedback from lung stretch receptors and paralyzed (pancuronium bromide 0.4 mg, i.v., maintained at $0.2 \mathrm{mg} / \mathrm{h}$ ). End-tidal $\mathrm{CO}_{2}$ was measured continuously (CapStar 100; CWE).

Nerve recording and stimulation. Rats were positioned nose-down in a stereotaxic frame, and the left phrenic, vagus, and renal nerves were dissected, tied, cut, and recorded using bipolar electrodes in warm mineral oil or silicon gel: phrenic nerve activity (PNA), vagal nerve activity (VNA), and renal sympathetic nerve activity (rSNA), respectively). Neurograms were amplified, bandpass filtered ( $100-3 \mathrm{kHz}, \mathrm{CWE})$, and sampled at $5 \mathrm{kHz}$ using a Power 1401 plus running Spike2 software (Cambridge Electronic Design).

In a subset of experiments, the left superior laryngeal nerve (SLN) was stimulated to induce fictive swallow. The nerve was isolated at the level of the carotid bifurcation, cut, and mounted on bipolar stimulating electrodes, and apneic threshold determined by stimulating at increasing intensities $(20 \mathrm{~Hz}, 0.2 \mathrm{~ms}$ pulses). In subsequent trials, the SLN was stimulated at 1.5 times apneic threshold.

Brain microinjection. Neurons in the IRt were acutely inhibited by bilateral pressure microinjections of the $\mathrm{GABA}_{\mathrm{A}}$ receptor agonist isoguvacine $(10 \mathrm{~mm}, 20-40 \mathrm{nl})$ mixed with pontamine sky blue $(\sim 2 \%)$ or fluorescent beads (Lumafluor, 1:2000 dilution). Microinjections were made $1.6 \mathrm{~mm}$ lateral, $1.2-1.5 \mathrm{~mm}$ rostral to obex and $2.4-2.5 \mathrm{~mm}$ deep to the brainstem surface. At the end of the experiments, rats were killed by sodium pentobarbitone overdose, transcardially perfused, and prepared for immunohistochemistry to confirm injection sites.

Data analysis and experimental protocols. Neurograms were rectified and smoothed ( $\tau=50 \mathrm{~ms}$ : PNA and VNA, $100 \mathrm{~ms}$ : rSNA). The peak-totrough amplitude of PNA, and the post-I recruitment of VNA and rSNA, were quantified from phrenic-triggered waveform averages. Peak post-I VNA and rSNA were measured with reference to their values at the onset of the post-I period, which could be unambiguously identified from an inflection in VNA that occurred at the end of the inspiratory period.

For quantification of effects of IRt inhibition on baseline respiratory and cardiovascular parameters, phrenic-triggered waveform averages were compiled from blocks of 10 respiratory cycles immediately before (baseline) and $30 \mathrm{~s}$ after unilateral or bilateral isoguvacine injection. The amplitudes of neurograms were normalized to baseline; other parameters (mean arterial pressure, heart rate, expiratory/inspiratory duration $\left[\mathrm{T}_{\mathrm{E}}\right.$ and $\mathrm{T}_{\mathrm{I}}$, respectively], and respiratory frequency) were measured over the same periods and analyzed without normalization. Significant effects were identified using nonparametric one-way ANOVA (Friedman test), followed, where indicated, by Dunn's multiple-comparison test to identify significantly different pairings (GraphPad, Prism 8).

The effects of acute hypoxemia and hypercapnia were compared in a separate cohort of animals. Following establishment of baseline recordings, animals were exposed to 1-2 min of acute hypercapnia or hypoxemia; respiratory parameters were allowed to recover for $\sim 20 \mathrm{~min}$; then gases were switched and the trial repeated so that each animal was exposed to both hypercapnia and hypoxemia. Hypercapnia was induced by ventilation with $5 \% \mathrm{CO}_{2}$ in $95 \%$ oxygen; hypoxemia by ventilation in room air (i.e., 21\%) (Saha et al., 2019). Initial experiments showed this reduced arterial $\mathrm{pO}_{2}$ from $494 \pm 11$ to $60 \pm 2 \mathrm{mmHg}(n=8)$. For quantification of neural responses to hypercapnia or hypoxemia, data were split into nine epochs of 10 respiratory cycles and phrenic-triggered averages of PNA, VNA, and rSNA were generated for each epoch, with the first beginning 10 cycles before gas administration. Measurements of peak-to-trough PNA amplitude and post-I VNA and rSNA were normalized such that the largest epoch measured in each animal became $100 \%$. The recruitment of nerve activities by hypercapnia versus hypoxemia were compared using repeated-measures two-way ANOVA; differences in activity evoked by each intervention were compared at each time point with the uncorrected Fisher's Least Significant Difference Test. Where differences in the recruitment of neural activity by hypercapnia/hypoxia were detected, their magnitudes were estimated by measuring area under the curve (AUC) of each series (i.e., the total activity summed across all epochs), compared between groups using paired $t$ test. Linear regression was performed to compare the covariance of post-I rSNA with VNA during hypercapnic and hypoxemic trials. $r^{2}$ values of responses to hypercapnic and hypoxemic trials were pooled between experiments and compared using paired $t$ test.

For quantification of responses to acute hypoxemia before and after bilateral IRt inhibition, PNA and post-I VNA and rSNA was measured as described above (although the duration of trials was shortened such that only 6 epochs were analyzed). After recovery from an initial hypoxemic trial, bilateral injections of isoguvacine were made into the IRt and hypoxemia repeated. Data from each epoch were normalized and analyzed as described above. In 3 cases, rSNA recordings were eliminated from analysis because of drift in baseline post-I amplitude between trials (i.e., the value of post-I rSNA before the first hypoxemic trial was substantially different from that measured after recovery from hypoxemia but before IRt isoguvacine microinjection).

The effects of IRt inhibition on fictive swallow and swallow-related apnea were assessed by comparing the number of clearly discernable vagal and phrenic bursts, respectively, observed during SLN stimulation, before and after unilateral isoguvacine injection. Statistically significant differences in SLN-evoked activity were assessed using Wilcoxon matched-pairs signed rank test. Autocorrelograms of rSNA and VNA during SLN stimulation were generated using Spike2 software. All data are presented as mean \pm SEM; the threshold for statistical significance was set at $p<0.05$.

\section{Results}

Distribution of ChAT-immunoreactive neurons in the intermediate reticular nucleus

We first examined the distribution of $\mathrm{ChAT}^{+}$neurons in the reticular formations of ChAT-cre mice and Lewis rats. A similar 

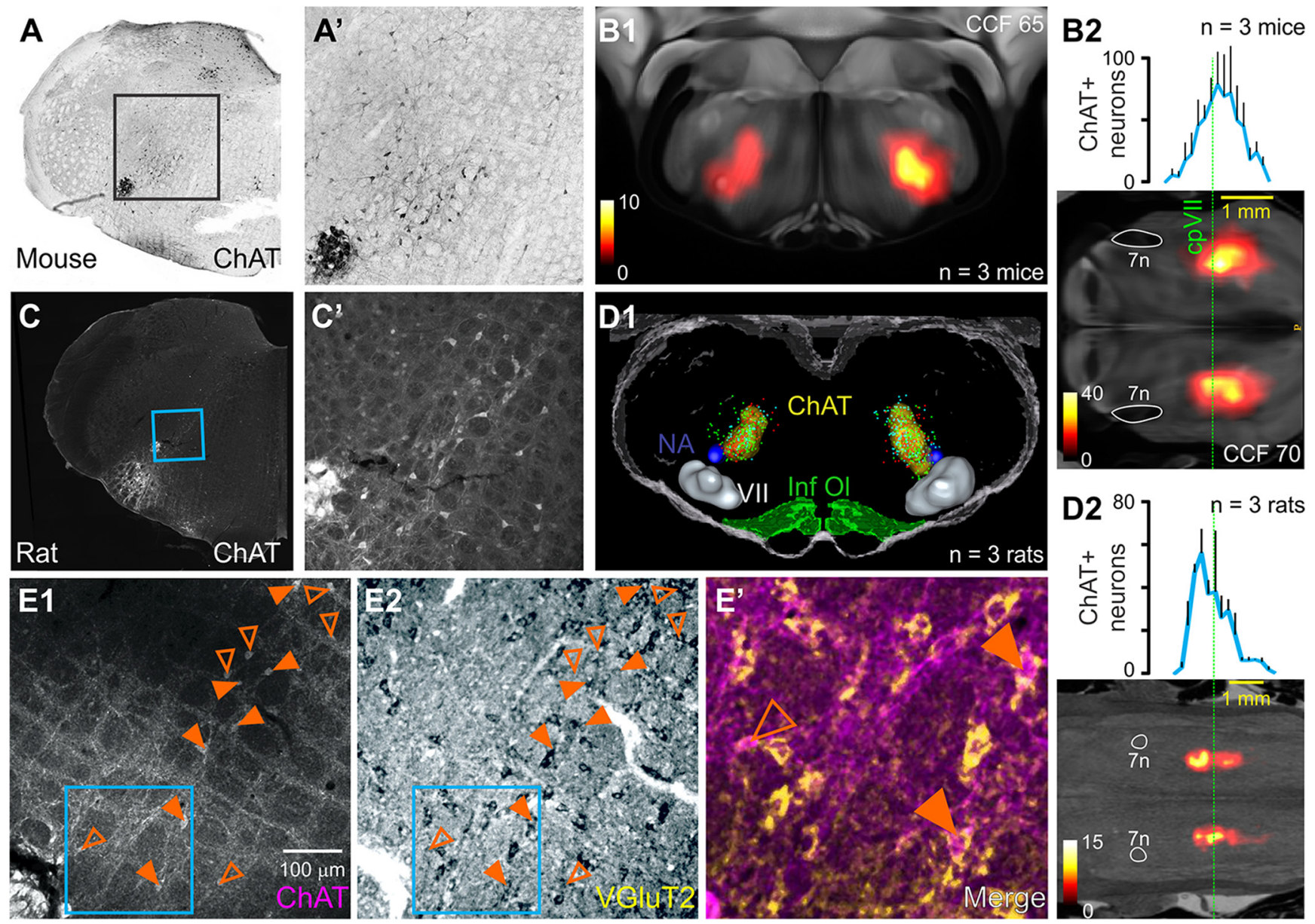

Figure 1. Distribution of ChAT-immunoreactive neurons in the IRt of the mouse and rat. $\boldsymbol{A}$, High magnification of boxed region in $\boldsymbol{A}^{\prime}$ : lightly immunoreactive neurons dorsomedial to nucleus ambiguus in the ChAT-cre mouse. $\boldsymbol{B 1}$, Heatmap represents the epicenter of the $\mathrm{ChAT}^{+}$population in a 100- $\mu \mathrm{m}$-thick virtual coronal section superimposed onto the corresponding plate of the Allen Brain Atlas (level 65 of the Common Coordinate Framework [CCF]). Average rostrocaudal distribution of $\mathrm{ChAT}^{+}$IRt neurons in the mouse is shown in the heatmap in $\boldsymbol{B 2}$ and graphed to the same scale at top. Dotted green line indicates the level of the caudal facial nucleus (cpVII). C, ChAT immunofluorescence in the rat IRt ( $\boldsymbol{C}^{\prime}$, inset). D1, Individual data from 3 rats (colored dots), with density maps

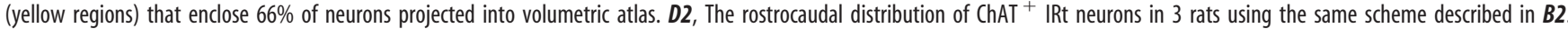

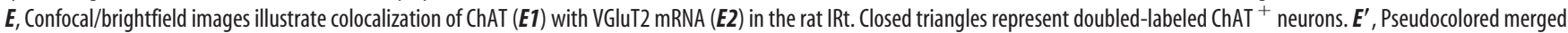
image of region indicated by blue box in E1, E2. Anatomical landmarks are nucleus ambiguus (NA), the facial nucleus (VII), inferior olive (Inf. Ol.), and facial nerve (7n).

pattern was observed regardless of species: weakly, $\mathrm{ChAT}^{+}$neurons were diffusely distributed within the IRt medial and dorsal to nucleus ambiguus (Fig. 1). In the mouse, the epicenter lay around Allen coordinates 180,65,70, corresponding to a position $150 \mu \mathrm{m}$ dorsal and $150 \mu \mathrm{m}$ medial to nucleus ambiguus, $\sim 150$ $\mu \mathrm{m}$ caudal to the facial nucleus (Fig. $1 A, B 1$ ), with $50 \%$ of neurons lying on average between 0 and $500 \mu \mathrm{m}$ caudal to the facial nucleus (Fig. 1B2; $n=3$ ). In the Lewis rat, $\mathrm{ChAT}^{+}$neurons were most densely aggregated $220 \mu \mathrm{m}$ medial and $350 \mu \mathrm{m}$ dorsal to nucleus ambiguus, slightly rostral to the caudal pole of the facial nucleus (Fig. 1C,D1), with $50 \%$ of neurons lying between on average $300 \mu \mathrm{m}$ rostral and $460 \mu \mathrm{m}$ caudal to the facial nucleus (Fig. 1D2; $n=3$ ). A density-mapped segmentation that enclosed $66 \%$ of labeled neurons spanned the IRt $\sim 1500 \mu \mathrm{m}$ in the rostrocaudal and $850 \mu \mathrm{m}$ in the dorsoventral planes (Fig. 1D1). Within the core of this region, $62 \pm 5 \%$ of $\mathrm{ChAT}^{+}$IRt neurons were also positive for VGluT2 mRNA (Fig. $1 E ; n=3$ ).

IRt inhibition reduces baseline post-I vagal and sympathetic activities

Guided by the anatomical data presented in Figure 1, we investigated whether inhibition of the region of the IRt that contained the highest density of $\mathrm{ChAT}^{+}$neurons diminished eupneic post-I activity or any other respiratory or cardiovascular parameters.

Bilateral inhibition of the IRt significantly inhibited the post-I components of VNA $(-65.3 \pm 9.2 \%$, Dunn's $p=0.0007)$ and rSNA $(-46.9 \pm 9.5 \%$, Dunn's $p=0.0016$; Fig. 2$)$. Effects were immediate and recovered over 5-10 min. IRt inhibition was also associated with a reduction in PNA $(-10.5 \pm 3.2 \%$, Dunn's $p=$ $0.015)$ and an increase in respiratory cycle frequency $(42.6 \pm 1.2$ vs $49.5 \pm 2.5 \mathrm{bpm}$, Dunn's $p=0.011$, equivalent to a $0.18 \pm 0.05 \mathrm{~s}$ reduction in respiratory cycle duration) due to a reduction in $\mathrm{T}_{\mathrm{E}}$ $(0.87 \pm 0.04$ vs $0.73 \pm 0.06 \mathrm{~s}$, Dunn's $p=0.0008)$, with no effect on $\mathrm{T}_{\mathrm{I}}$ observed $(0.52 \pm 0.01$ vs $0.53 \pm 0.03 \mathrm{~s}$, Friedman $p=0.4)$. Bilateral IRt inhibition also evoked a small but significant effect on heart rate ( $460 \pm 6$ vs $456 \pm 7 \mathrm{bpm}$, Dunn's $p=0.022)$ and no significant effect on mean arterial pressure $(62 \pm 5$ vs $58 \pm 4$ $\operatorname{mmHg}$, Friedman $p=0.3)$ or mean rSNA $(-0.8 \pm 2.2 \%$, Fried$\operatorname{man} p=0.19)$. We therefore conclude that the ongoing activity of neurons in the IRt contributes to eupneic respiratory and sympathetic post-I activities without exerting significant control on other respiratory or cardiovascular parameters. 

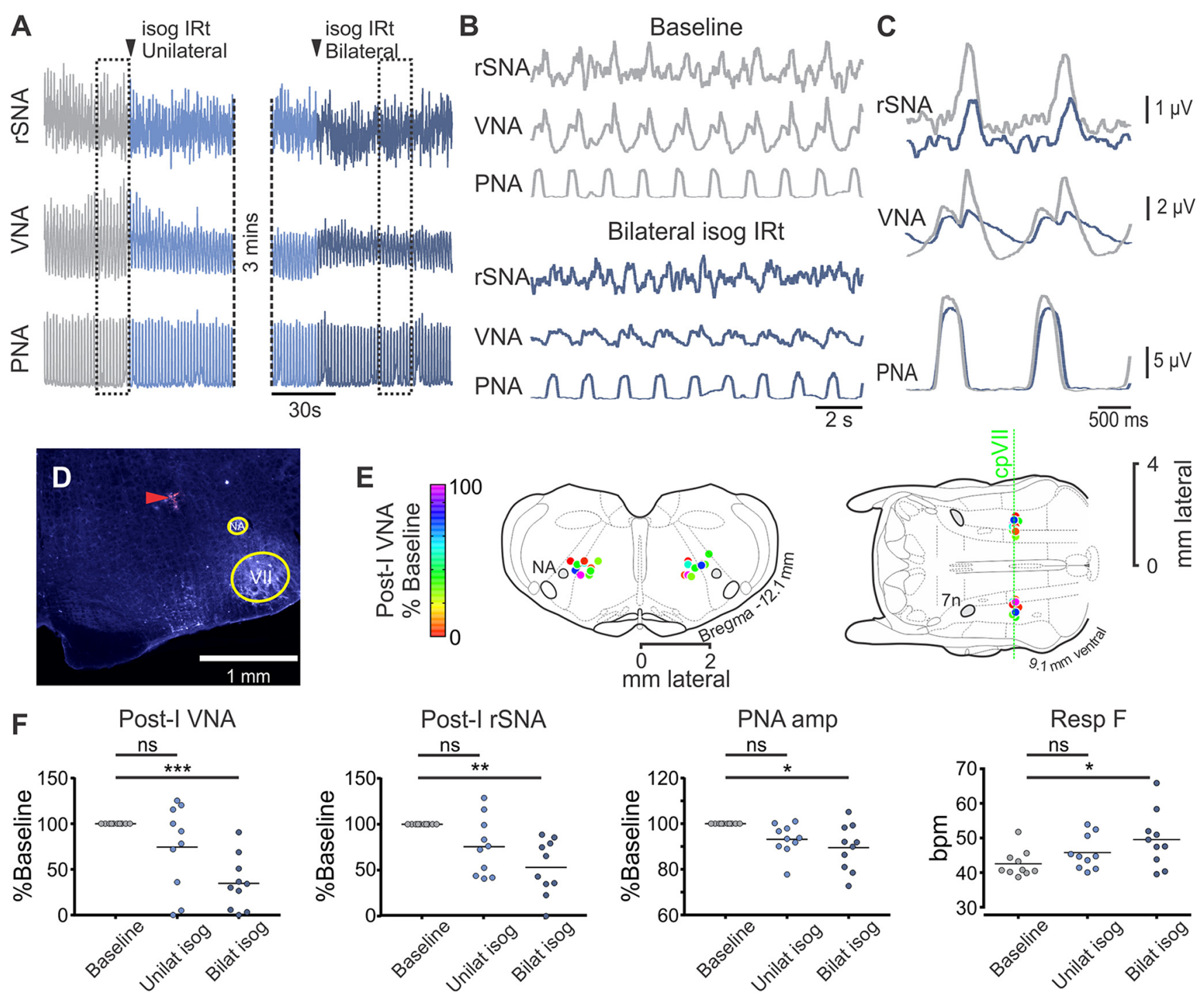

Figure 2. IRt inhibition attenuates eupneic post-I activity. $\boldsymbol{A}$, Experimental recording from urethane-anesthetized Lewis rat; bilateral microinjections of isoguvacine (isog) were made into the IRt. $\boldsymbol{B}$, Expanded view of boxed regions from $\boldsymbol{A}$ and corresponding phrenic-trigged averages in $\boldsymbol{C}$ show attenuated post-I VNA and rSNA without significant effects on respiratory pattern. $\boldsymbol{D}$, Histological section showing representative injection site marked by fluorescent beads (red arrowhead). $\boldsymbol{E}$, Composite images represent injection sites from multiple experiments mapped onto corresponding coronal (left) and horizontal (right) atlas plates, color-coded to indicate effect of bilateral isoguvacine on post-I VNA. $\boldsymbol{F}$, Pooled data indicate that bilateral IRt inhibition significantly reduces post-I VNA, rSNA, and PNA, and increases respiratory frequency. Dunn's post-test: ns, not significant; ${ }^{*} p<0.05 ;{ }^{* *} p<0.01 ;{ }^{* * *} p<0.001$.

Post-I activity is stimulated by acute hypoxemia

We next compared the effects of hypercapnia and hypoxemia on respiratory dynamics and post-I VNA and rSNA in urethaneanesthetized vagotomized rats (Fig. 3). Hypercapnic and hypoxemic trials maximally increased phrenic nerve amplitude over similar time courses and to similar degrees (AUC $706 \pm 22$ vs $690 \pm 21$ a.u., respectively, $n=8$, paired $t$ test $p=0.6$ ), but hypoxemia produced significantly more post-I activity in both vagal and sympathetic outputs compared with hypercapnia (twoway ANOVA interaction: VNA: $F_{(8,112)}=4.8, n=8, p<0.0001$; rSNA: $F_{(8,112)}=27.8, n=8, p<0.0001$, significantly different time points indicated in Figure $3 D)$. Measured by AUC, this effect manifested as a $49 \pm 22 \%$ greater increase in post-I VNA ( $423 \pm$ 66 vs $541 \pm 44$ a.u., $n=8$, paired $t$ test $p=0.003)$ and $75 \pm 20 \%$ greater increase in post-I rSNA ( $287 \pm 27$ vs $465 \pm 19$ a.u., $n=8$, paired $t$ test $p=0.004$ ) in response to hypoxemia compared with hypercapnia. Interestingly, we also observed differences in the relationship between post-I VNA and post-I rSNA under these conditions: hypoxemia resulted in progressive recruitment of both outputs, whereas hypercapnia resulted in recruitment of post-I VNA only (Fig. 3C,D). This relationship was investigated by linear regression of post-I rSNA plotted against simultaneously recorded post-I VNA (Fig. $3 E$ ). During hypoxemic trials, post-I VNA and post-I rSNA were coactivated (linear regression: $\left.r^{2}=0.84 \pm 0.05, p=0.002 \pm 0.001, n=8\right)$; this effect was not apparent during hypercapnia $\left(r^{2}=0.26 \pm 0.09, p=0.37 \pm 0.13\right)$. We conclude that hypoxemia stimulates post-I activity with particular efficacy compared with hypercapnia, and that post-I activities in laryngeal and autonomic outputs may be independently controlled to some extent.

\section{Post-I responses to acute hypoxemia persist following inhibition of the IRt}

We next examined whether activity in the IRt is necessary for the hypoxemic recruitment of post-I in vagal and sympathetic outputs (Fig. 4). Bilateral microinjection of isoguvacine selectively reduced the magnitudes of post-I VNA and rSNA, but not PNA responses to acute hypoxemia (two-way ANOVA isoguvacine: 


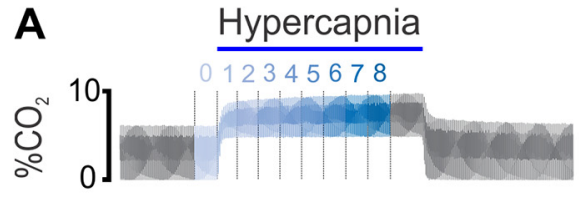

rSNA

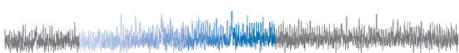

VNA

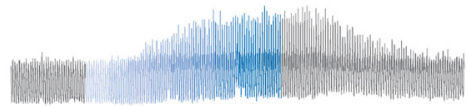

PNA

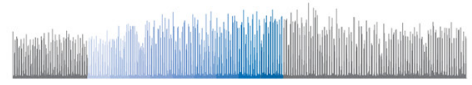

D

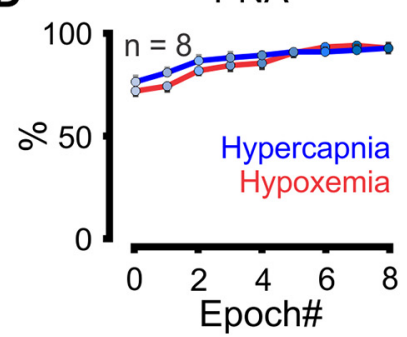

Hypoxemia
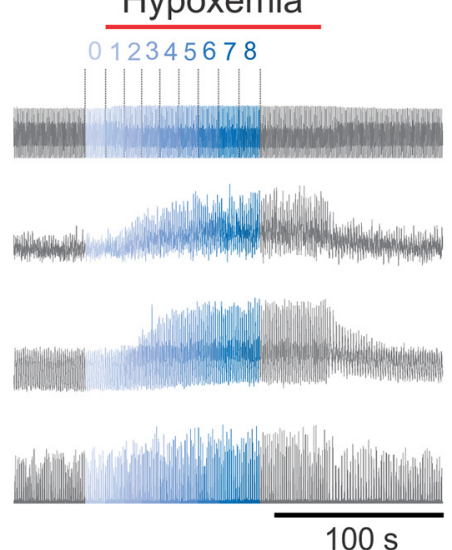

B

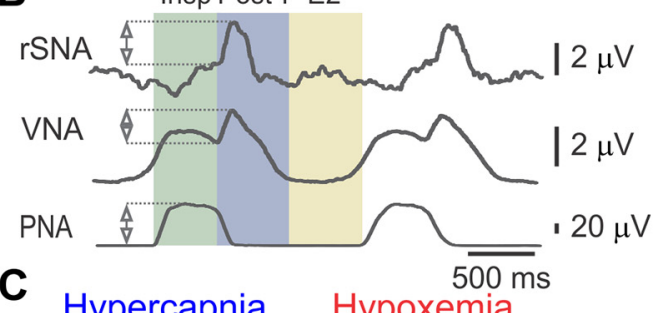

C Hypercapnia

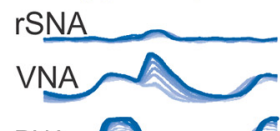

Hypoxemia

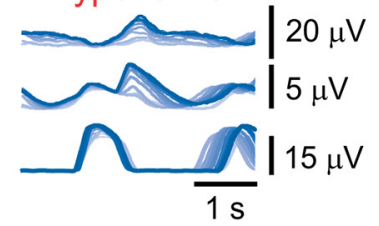

rSNA

E
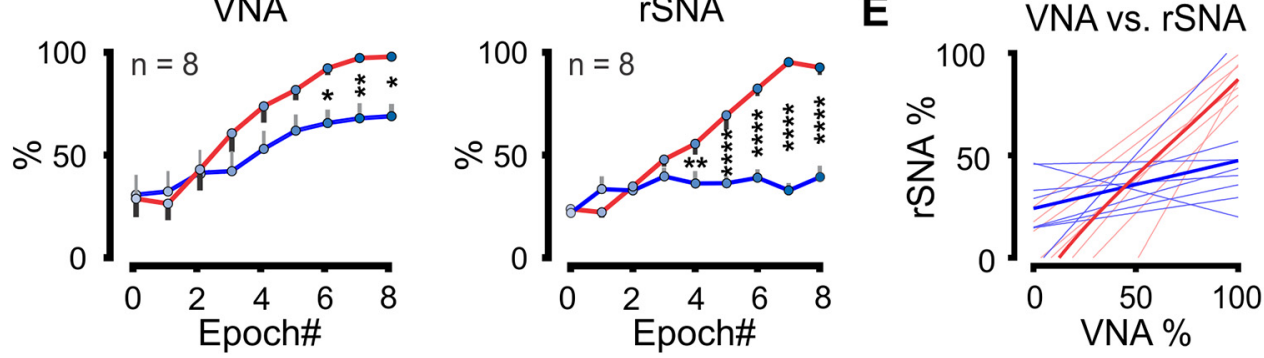

Figure 3. Hypoxemia and hypercapnia differentially activate post-I activities. $A$, Recording from urethane-anesthetized Lewis rat illustrating increases in PNA, post-I VNA, and post-I rSNA in response to acute hypercapnia and hypoxemia. $\boldsymbol{B}$, Phrenic-triggered waveform averages were used to quantify respiratory-related nerve activities. Colored rectangles represent respiratory phases. Insp, Inspiratory; E2, late expiratory. Double-headed arrows indicate quantified parameters. C, Both stimuli progressively recruited PNA, but post-I VNA and rSNA were differentially activated by hypoxemia; phrenic-triggered averages correspond to color-coded epochs indicated in $\boldsymbol{A}$. D, Pooled data compare hypercapnic and hypoxemic recruitment of PNA, VNA, and rSNA. Significant pairings were identified using Fisher's Least Significant Difference Test. $\boldsymbol{E}$, Linear regression shows parallel recruitment of rSNA with VNA during hypoxemic trials $\left(r^{2}=0.84 \pm 0.05, p=0.002\right.$, red lines), but not hypercapnic trials ( $p>0.3$, blue lines). Light red or blue represents individual trials. Bold represents average responses. ${ }^{*} p<0.05 .{ }^{* *} p<0.01 .{ }^{* * *} p<0.0001$.

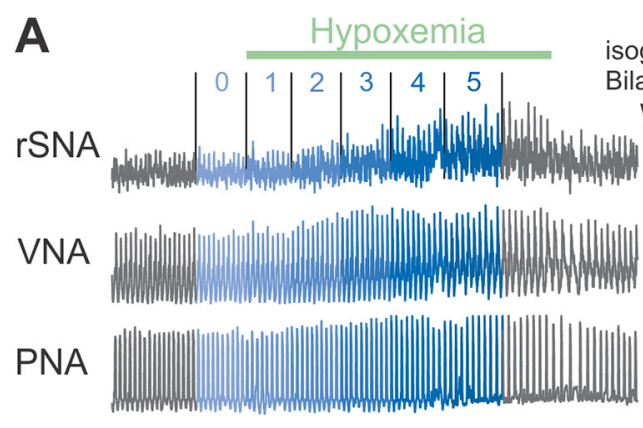

C

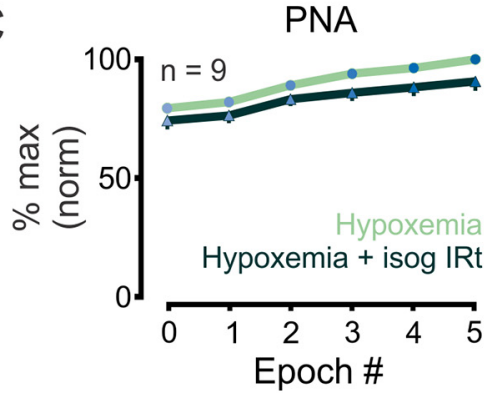

isog IRt

Bilateral
Hypoxemia + isog IRt

\section{,}
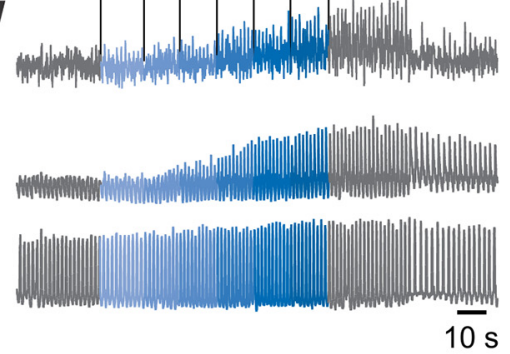

VNA

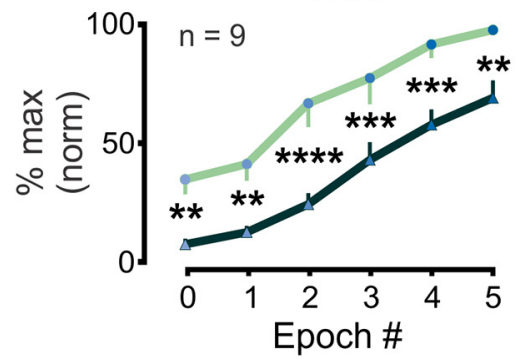

B Hypoxemia Hypoxemia + isog IRt
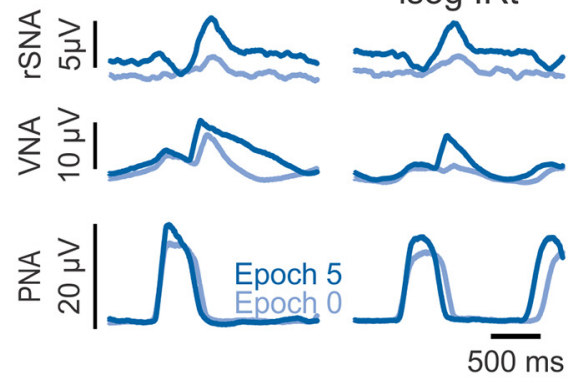

rSNA

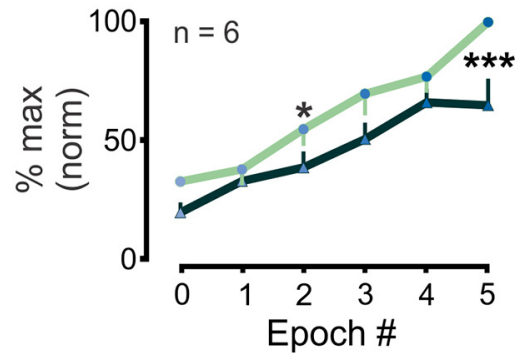

Figure 4. Hypoxemic recruitment of post-I activity persists after IRt inhibition. $\boldsymbol{A}$, Urethane-anesthetized rats were exposed to brief hypoxemia. Following recovery of respiratory parameters, bilateral microinjections of isoguvacine were made into the IRt and rats reexposed to hypoxemia. $\boldsymbol{B}$, Phrenic-triggered waveform averages from the same experiment showing rSNA, VNA, and PNA responses to acute hypoxemia before (left) and after (right) bilateral IRt inhibition. C, Pooled data indicate that IRt inhibition reduces, but does not block, hypoxemic recruitment of post-I VNA or rSNA and exerts no significant effect on PNA. Significant pairings were identified using Fisher's Least Significant Difference Test: * $p<0.05$; ${ }^{* *} p<0.01 ;{ }^{* * *} p<0.001 ;{ }^{* * *} p<0.0001$. 


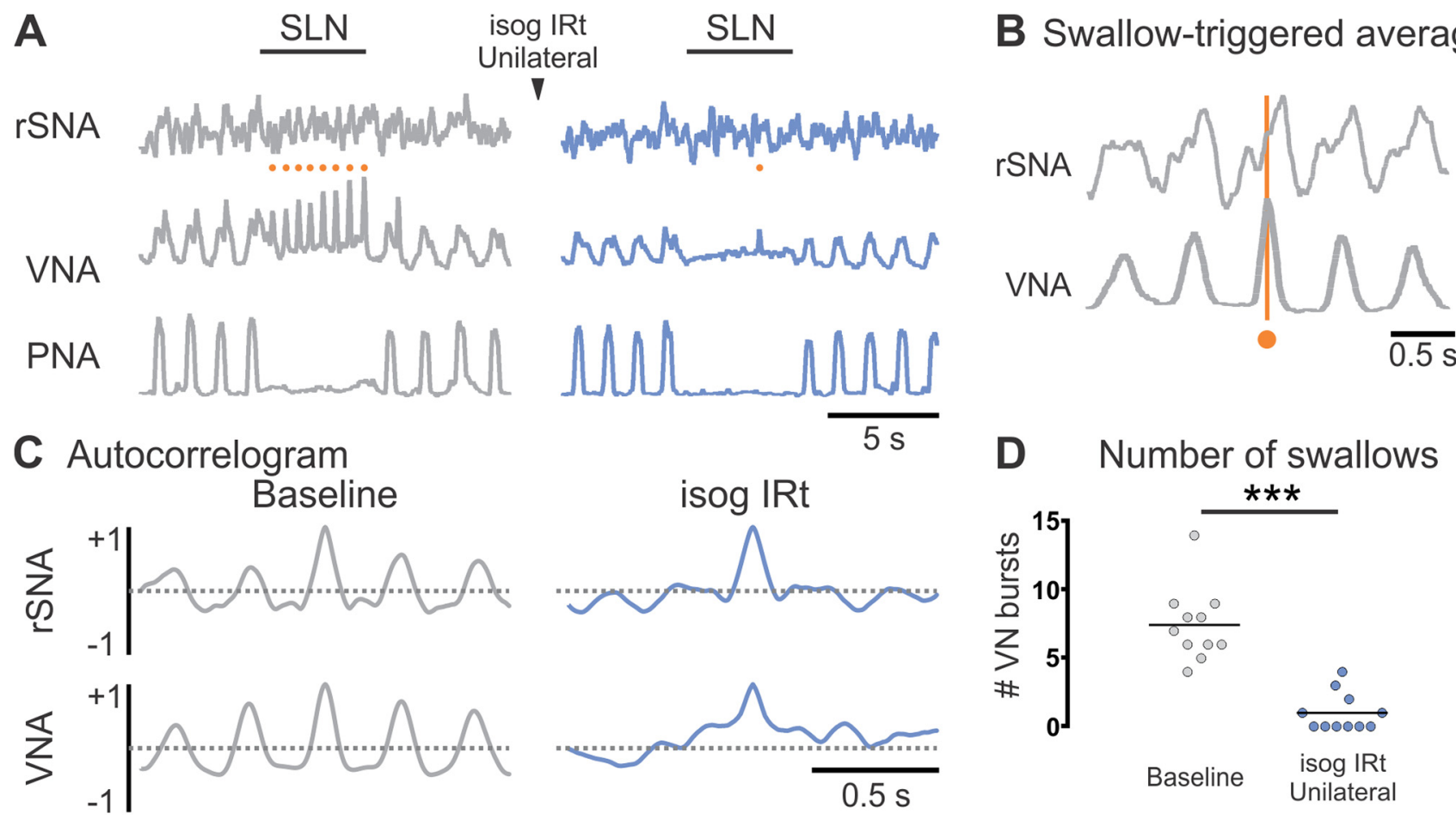

Figure 5. IRt activity is required for coordination of fictive swallow. $A$, Under baseline conditions ( gray), electrical stimulation of the SLN evoked apnea and rhythmic VNA (fictive swallows, orange circles). $\boldsymbol{B}$, Swallow-triggered averages of rSNA and ( $\boldsymbol{C}$ rSNA autocorrelograms reveal swallow-related activity. Inhibition of the ipsilateral IRt reduced the number of fictive swallows $(\boldsymbol{A}$, blue; pooled data in $\boldsymbol{D}$ ) (significant differences identified using Wilcoxon matched-pairs signed rank test) and disrupted swallow-locked rSNA activity ( $\boldsymbol{C}$, blue) without inhibiting the apneic component of the response to SLN stimulation. ${ }^{* * *} p<0.001$.

VNA: $F_{(1,16)}=18.99, n=9, p=0.0005$; rSNA: $F_{(1,10)}=5.607$, $n=6, p=0.039$; PNA: $F_{(1,16)}=3.85, n=9, p=0.067$; Figure $4 C$ ) but did not alter the gain of hypoxemic responses (two-way ANOVA interaction: VNA: $p=0.56$; rSNA: $p=0.07$; PNA: $p=$ 0.32 ), resulting in a rightwards shift in their stimulus-response curves. Similarly, IRt inhibition reduced the AUC of post-I vagal responses ( $343 \pm 35$ vs $176 \pm 22$ a.u., paired $t$ test $p=0.0039, n=$ $9)$, but had no significant effect on the AUC of post-I rSNA $(305 \pm 24$ vs $230 \pm 28, p=0.16, n=6)$ or PNA ( $451 \pm 6$ vs $416 \pm$ $17, p=0.13)$. Thus, IRt inhibition does not completely block the potent recruitment of post-I activity evoked by acute hypoxic stimuli.

\section{The IRt is a critical relay for fictive swallow}

Under baseline conditions, supramaximal SLN stimulation evoked apnea and gradual ramp activation of VNA superimposed with rapid rhythmic bursts ("fictive swallows": Fig. 5) as previously described (Sun et al., 2011). In 10 of 11 experiments, we also detected swallow-locked oscillations in rSNA that were apparent in swallow-triggered waveform averages (Fig. 5B) and nerve autocorrelograms generated during SLN stimulation (Fig. 5C), showing that, like eupneic post-I activity, the output of the swallow CPG is transmitted to both motor and vasomotor outputs.

Ipsilateral IRt inhibition reduced the number of swallows evoked during SLN stimulation from $7.5 \pm 0.8$ to $1.0 \pm 0.4$ (Wilcoxon $p=0.001$; Fig. $5 D$ ) without blocking the apneic component of the response $(0.5 \pm 0.3$ vs $0.8 \pm 0.3$ phrenic bursts/ trial, Wilcoxon $p=0.69$ ) or ramp activation of VNA. IRt inhibition also abolished the rhythmic activities evident in rSNA and VNA autocorrelograms. These findings indicate that IRt neurons play a critical role in the transmission of rhythmic motor and vasomotor components of fictive swallow, but do not mediate the apneic component of the swallowing pattern.

\section{Discussion}

The rat medulla contains a population of lightly $\mathrm{ChAT}^{+}$neurons, many of which are glutamatergic, which is diffusely scattered through the IRt and most densely distributed immediately dorsomedial to nucleus ambiguus at the level of the facial nucleus. Consistent with previous surveys in the rat (Tago et al., 1989), we failed to replicate the restricted clustering of ChAT neurons reported to define the spatial extent of the mouse PiCo (e.g., Fig. 1B) (Anderson et al., 2016). A similar pattern of diffuse ChAT immunoreactivity also predominated in our examination of the mouse, and Allen Brain Atlas Experiments 252 (ChAT mRNA) and 100138934 (Chat-IRES-Cre-neo;Ai32(RCL-ChR2(H134R)_ EYFP), EGFP mRNA). Nonetheless, under baseline conditions, bilateral inhibition of this region robustly inhibited post-I vagal and sympathetic nerve activities; it is unlikely that these effects were mediated by direct inhibition of nearby sympathetic premotor or vagal motoneurons, as mean rSNA and the inspiratory component of VNA were unchanged. These findings suggest that neurons in the region of the IRt that contains the highest density of $\mathrm{ChAT}^{+}$neurons are closely involved in eupneic laryngeal and vasomotor post-I activities.

IRt inhibition caused small reductions in PNA and $\mathrm{T}_{\mathrm{E}}$, resulting in increased respiratory frequency, but had no effect on $\mathrm{T}_{\mathrm{I}}$. This indicates that neurons in the IRt do not contribute to the "inspiratory off-switch," a barrage of post-I inhibition that terminates activity in inspiratory neurons and truncates the inspiratory phase of the respiratory cycle (Richter, 1982; Rybak et al., 2007). These effects contrast with those observed following loss- 


\section{A Eupnea}

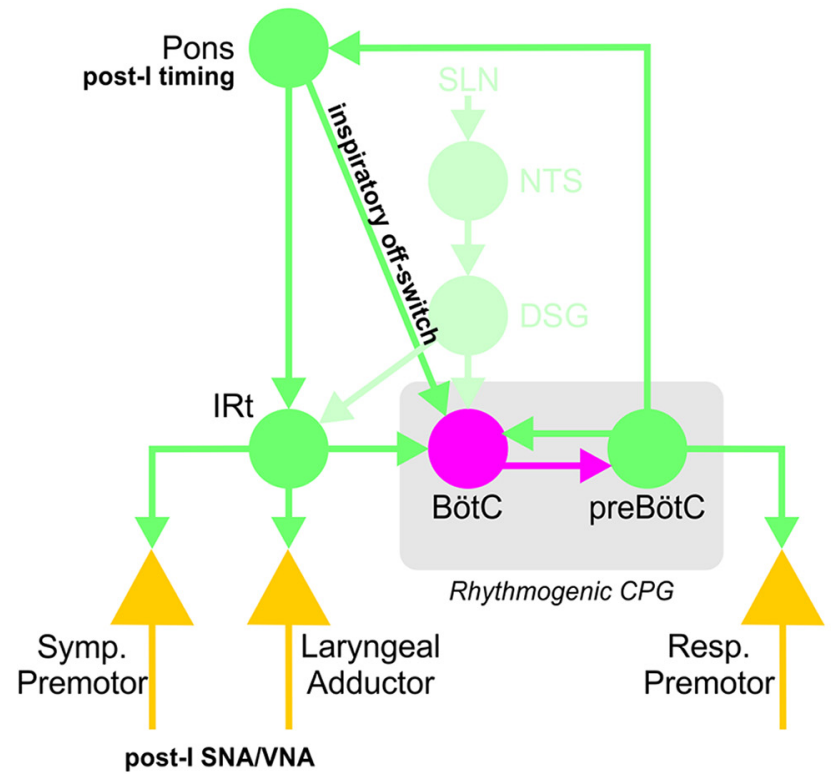

\section{B Swallow}

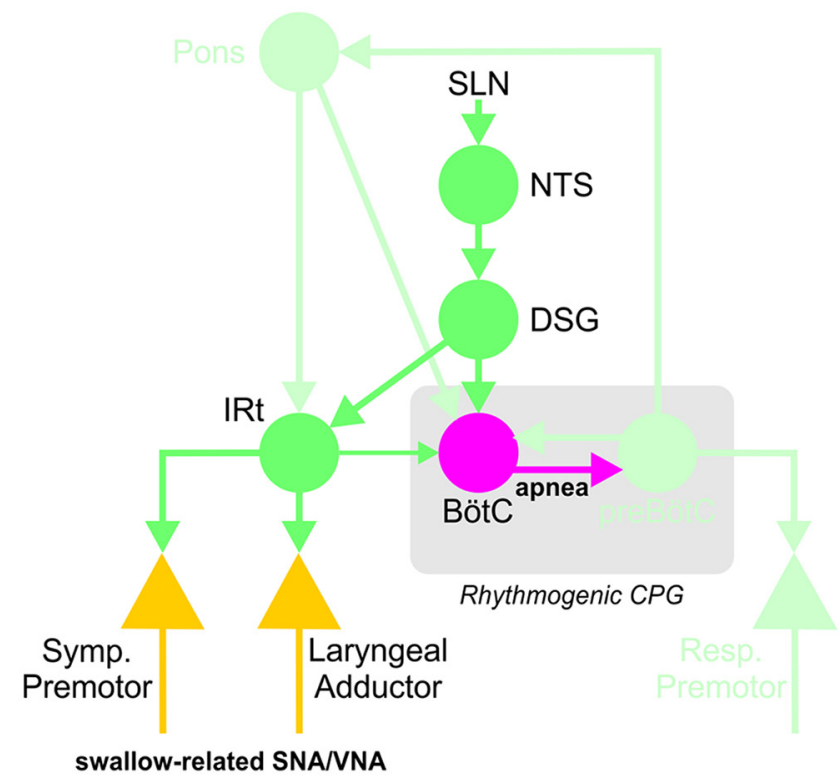

Figure 6. Simplified schematic diagram illustrating potential integration of IRt with respiratory and swallowing network components. A, During eupnea, post-I timing originates in the pons, recruiting post-I activities in laryngeal motor and sympathetic premotor outputs via relay in the IRt. Excitatory post-I drive from the pons also activates inhibitory BötC neurons that truncate inspiration, the so-called "inspiratory off-switch." B, Stimulation of laryngeal afferent fibers in the SLN excites second-order NTS neurons that trigger swallow via activation of the DSG. DSG activation results in powerful recruitment of BötC neurons, arresting respiratory rhythm, and activation of the ventral swallowing group located in the IRt, which transmits rhythmic swallow-related activity to respiratory and sympathetic outputs. Bright green represents excitatory components. Magenta represents inhibitory connections. Pastel green represents inactive components. Yellow triangles represent output neurons. Symp. Premotor, RVLM sympathetic premotor neurons; Resp. Premotor, respiratory premotor circuits in the rostral and caudal ventral respiratory groups.

of-function in the pons, BötC, or nucleus of the solitary tract (NTS), which also abolish post-I motor/autonomic outputs and have variable effects on $\mathrm{T}_{\mathrm{E}}$ but are associated with the development of an apneustic breathing pattern characterized by prolonged $\mathrm{T}_{\mathrm{I}}$ (Baekey et al., 2008, 2010; Burke et al., 2010; CostaSilva et al., 2010; Bautista and Dutschmann, 2016; Farmer et al., 2016). The simplest explanation for this discrepancy is that the post-I synaptic drive evident in respiratory pattern-generating interneurons in the $\mathrm{BötC}$ and preBötC does not originate in the IRt: the pontine respiratory groups are strong candidate sources (Dutschmann and Herbert, 2006; Dick et al., 2009).

Acute hypoxemia reduced arterial $\mathrm{pO}_{2}$ to $\sim 60 \mathrm{mmHg}$, equivalent to levels observed in chronic obstructive pulmonary disease or sleep apnea (Fletcher et al., 1987), and potently recruited post-I activity. As expected, bilateral IRt inhibition following recovery from initial hypoxemic trials reduced baseline post-I activity but did not significantly disrupt post-I recruitment by subsequent hypoxemia. These findings suggest that IRt neurons are not the only source of post-I drive to these outputs, and that mechanisms that are relatively quiescent in eupnea may be recruited under hypoxemia. Such hypoxia-sensitive post-I neurons may reside elsewhere, or could simply be in parts of the IRt that lie beyond the spread of the injections used in the current study. The mapping of functionally identified post-I IRt neurons in vivo and confirmation of their putative $\mathrm{ChAT}^{+} \mathrm{VGLUT2}^{+}$phenotype should be a priority for future investigations; we predict that neurons responsible for generating post-I activity will be exquisitely sensitive to low oxygen.

Although our data unambiguously support a role for IRt neurons in the coordination of eupneic post-I motor/vasomotor activities, it seems their participation is not necessarily necessary (Yoshihara and Yoshihara, 2018) for the recruitment of post-I activity during hypoxemia, and that their contribution is limited to the motor/vasomotor, but not central, manifestations of post-I activity. We wondered whether, instead of representing a distinct CPG for post-I activity, neurons in the IRt may instead serve as a premotor relay that transmits convergent input from closely coordinated respiratory and nonrespiratory CPGs, such as swallow (and perhaps sneeze and cough), which share common motor outputs and are also recruited in the post-I period (Bartlett and Leiter, 2011; Sun et al., 2011; Bautista and Dutschmann, 2014; Dutschmann et al., 2014).

Unilateral IRt inhibition abolished the rhythmic vagal (and previously unreported sympathetic) elements of fictive swallow without disrupting apnea. This effect can be reliably attributed to inhibition of IRt neurons and not nearby BötC neurons, many of which are post-I neurons that become phasically active during fictive swallow (Saito et al., 2003), as deliberate inhibition of BötC has the converse effect, blocking apnea but not swallow (Sun et al., 2011). Thus, in addition to containing neurons that contribute to eupneic post-I activity in laryngeal and vasomotor nerves, the IRt also contains neurons that transmit rhythmic swallowrelated activity to the same outputs. We argue that such neurons likely represent a premotor output and not the swallowing CPG, as the apneic component of the swallowing response persisted.

These observations accord well with the consensus model of the swallowing CPG, in which stimulation of laryngeal sensory fibers robustly activates neurons in a dorsal swallowing group (DSG) adjacent to the NTS (for review, see Jean, 2001; Jean and Dallaporta, 2013). The activation of this group is required for both the apneic and motor components of swallow (Bautista and Dutschmann, 2014), and it contains many neurons that burst in phase with swallow and project to the IRt immediately dorsomedial to nucleus ambiguus (Kessler and Jean, 1985; Gestreau et al., 1996; Sugiyama et al., 2011). This region contains the ventral swallowing group, a population of neurons that also burst in 
phase with swallow but respond to orthodromic SLN stimulation at longer latencies than DSG neurons and are therefore thought to represent a downstream relay that transmits swallowing motor patterns generated in the DSG to oropharyngeal outputs (Kessler and Jean, 1985; Amri et al., 1990; Ezure et al., 1993; Umezaki et al., 1998). The axonal trajectories of ventral swallowing neurons suggest innervation of motor pools involved in the oropharyngeal phase of swallow (Amri and Car, 1988; Ezure et al., 1993; Ono et al., 1998), and these findings are supported by connectomic studies that show that rostral IRt neurons form monosynaptic connections with motoneurons that innervate muscles involved in swallowing, such as the tongue and masseter (Stanek et al., 2014), and with RVLM sympathetic premotor neurons (Dempsey et al., 2017), but not respiratory pump muscles, such as the diaphragm (Wu et al., 2017). IRt neurons are therefore well placed to transmit drive to motor/vasomotor outputs that exhibit both post-I and swallowing activity.

Our data indicate that neurons in the IRt are involved in the transmission of post-I activity and are indispensable for the transmission of swallowing motor patterns to the same outputs, and so a key question for future investigation is whether post-I and swallowing IRt neurons are distinct classes or whether overlapping populations of neurons participate in both functions. The available evidence suggests that the former is more likely: post-I activity is an ongoing phenomenon, and yet ventral swallowing neurons are not generally spontaneously active (Kessler and Jean, 1985; Ezure et al., 1993) and, when active, rarely exhibit a post-I firing pattern (Ezure et al., 1993; Ono et al., 1998). However, it is not clear that there are any "nonswallowing" post-I neurons in this region either; although plentiful in the BötC, the evidence for IRt neurons with the predicted post-I firing pattern in vivo is largely anecdotal (Oku et al., 1994).

Our experimental design necessitated simultaneous access to multiple motor and sensory nerves, which was only feasible in the rat. Although post-I activity is highly conserved, and so the mechanisms that underlie its generation are presumably similar between species, the configuration of respiratory-sympathetic coupling varies considerably, with the post-I component dominating in the rat but not the cat, pig, or human (Häbler et al., 1994). Consequently, the potential contribution made by the IRt to respiratory-sympathetic coupling in other species cannot be extrapolated from the current study. When comparing our results to the excellent study by Anderson et al. (2016), a second consideration is differences in the spread of microinjected drugs. Our 20-40 nl isoguvacine injections probably influenced neurons within $\sim 350 \mu \mathrm{m}$ of the injection site (Burke et al., 2010). This has the advantage that isoguvacine would not have encroached into neighboring motor, sympathetic, or respiratory groups, but probably only reached a subset of $\mathrm{ChAT}^{+}$neurons, which are diffusely distributed within the IRt. In contrast, Anderson et al. (2016) used $250 \mathrm{nl}$ of somatostatin/DAMGO in the mouse, and therefore would have silenced a much large fraction of putative post-I neurons while risking off-target effects.

In conclusion, we are confident that IRt neurons are involved in the transmission of post-I activity to laryngeal adductor and vasomotor nerves. However, the absence of any significant effect of IRt inhibition on $\mathrm{T}_{\mathrm{I}}$ undermines the notion that these neurons are a critical component of a post-I CPG, whereas the finding that IRt inhibition also blocks the motor/vasomotor components of fictive swallow suggests involvement in a range of functions. As such, conceptualizing this cell group as a static and functionally dedicated post-I complex seems premature. One possibility is that the IRt represents a site of integration for motor and auto- nomic components of diverse respiratory and para-respiratory patterns, such as post-I and swallow, that are generated elsewhere (e.g., post-I pontine neurons and the DSG respectively; Fig. 6).

\section{References}

Alheid GF, McCrimmon DR (2008) The chemical neuroanatomy of breathing. Respir Physiol Neurobiol 164:3-11.

Amri M, Car A (1988) Projections from the medullary swallowing center to the hypoglossal motor nucleus: a neuroanatomical and electrophysiological study in sheep. Brain Res 441:119-126.

Amri M, Car A, Roman C (1990) Axonal branching of medullary swallowing neurons projecting on the trigeminal and hypoglossal motor nuclei: demonstration by electrophysiological and fluorescent double labeling techniques. Exp Brain Res 81:384-390.

Anderson TM, Garcia AJ 3rd, Baertsch NA, Pollak J, Bloom JC, Wei AD, Rai KG, Ramirez JM (2016) A novel excitatory network for the control of breathing. Nature 536:76-80.

Baekey DM, Dick TE, Paton JF (2008) Pontomedullary transection attenuates central respiratory modulation of sympathetic discharge, heart rate and the baroreceptor reflex in the in situ rat preparation. Exp Physiol 93:803-816.

Baekey DM, Molkov YI, Paton JF, Rybak IA, Dick TE (2010) Effect of baroreceptor stimulation on the respiratory pattern: insights into respiratorysympathetic interactions. Respir Physiol Neurobiol 174:135-145.

Bartlett D, Leiter JC (2011) Coordination of breathing with nonrespiratory activities. In: Comprehensive physiology. New York: Wiley.

Bautista TG, Dutschmann M (2014) Ponto-medullary nuclei involved in the generation of sequential pharyngeal swallowing and concomitant protective laryngeal adduction in situ. J Physiol 592:2605-2623.

Bautista TG, Dutschmann M (2016) The role of the Kölliker-Fuse nuclei in the determination of abdominal motor output in a perfused brainstem preparation of juvenile rat. Respir Physiol Neurobiol 226:102-109.

Bautista TG, Burke PG, Sun QJ, Berkowitz RG, Pilowsky PM (2010) The generation of post-inspiratory activity in laryngeal motoneurons: a review. Adv Exp Med Biol 669:143-149.

Bjerke IE, Øvsthus M, Andersson KA, Blixhavn CH, Kleven H, Yates SC, Puchades MA, Bjaalie JG, Leergaard TB (2018) Navigating the murine brain: toward best practices for determining and documenting neuroanatomical locations in experimental studies. Front Neuroanat 12:82.

Burguet J, Andrey P (2014) Statistical comparison of spatial point patterns in biological imaging. PLoS One 9:e87759.

Burke PG, Abbott SB, McMullan S, Goodchild AK, Pilowsky PM (2010) Somatostatin selectively ablates post-inspiratory activity after injection into the Bötzinger complex. Neuroscience 167:528-539.

Costa-Silva JH, Zoccal DB, Machado BH (2010) Glutamatergic antagonism in the NTS decreases post-inspiratory drive and changes phrenic and sympathetic coupling during chemoreflex activation. J Neurophysiol 103: 2095-2106.

Del Negro CA, Funk GD, Feldman JL (2018) Breathing matters. Nat Rev Neurosci 19:351-367.

Dempsey B, Le S, Turner A, Bokiniec P, Ramadas R, Bjaalie JG, Menuet C, Neve R, Allen AM, Goodchild AK, McMullan S (2017) Mapping and analysis of the connectome of sympathetic premotor neurons in the rostral ventrolateral medulla of the rat using a volumetric brain atlas. Front Neural Circuits 11:9.

Dick TE, Baekey DM, Paton JF, Lindsey BG, Morris KF (2009) Cardiorespiratory coupling depends on the pons. Respir Physiol Neurobiol 168: $76-85$.

Dick TE, Hsieh YH, Dhingra RR, Baekey DM, Galán RF, Wehrwein E, Morris KF (2014) Cardiorespiratory coupling: common rhythms in cardiac, sympathetic, and respiratory activities. Prog Brain Res 209:191-205.

Dutschmann M, Herbert H (2006) The Kölliker-Fuse nucleus gates the postinspiratory phase of the respiratory cycle to control inspiratory offswitch and upper airway resistance in rat. Eur J Neurosci 24:1071-1084.

Dutschmann M, Paton JF (2002) Glycinergic inhibition is essential for coordinating cranial and spinal respiratory motor outputs in the neonatal rat. J Physiol 543:643-653.

Dutschmann M, Jones SE, Subramanian HH, Stanic D, Bautista TG (2014) The physiological significance of postinspiration in respiratory control. Prog Brain Res 212:113-130.

Ezure K, Oku Y, Tanaka I (1993) Location and axonal projection of one type of swallowing interneurons in cat medulla. Brain Res 632:216-224. 
Farmer DG, Dutschmann M, Paton JF, Pickering AE, McAllen RM (2016) Brainstem sources of cardiac vagal tone and respiratory sinus arrhythmia. J Physiol 594:7249-7265.

Farmer DG, Pracejus N, Dempsey B, Turner A, Bokiniec P, Paton JFR, Pickering AE, Burguet J, Andrey P, Goodchild AK, McAllen RM, McMullan S (2019) On the presence and functional significance of sympathetic premotor neurons with collateralized spinal axons in the rat. J Physiol 597:3407-3423.

Feldman JL, Del Negro CA, Gray PA (2013) Understanding the rhythm of breathing: so near, yet so far. Annu Rev Physiol 75:423-452.

Fletcher EC, Miller J, Divine GW, Fletcher JG, Miller T (1987) Nocturnal oxyhemoglobin desaturation in COPD patients with arterial oxygen tensions above $60 \mathrm{~mm} \mathrm{Hg}$. Chest 92:604-608.

Gestreau C, Milano S, Bianchi AL, Grélot L (1996) Activity of dorsal respiratory group inspiratory neurons during laryngeal-induced fictive coughing and swallowing in decerebrate cats. Exp Brain Res 108:247-256.

Guyenet PG, Darnall RA, Riley TA (1990) Rostral ventrolateral medulla and sympathorespiratory integration in rats. Am J Physiol 259:R1063-R1074.

Häbler HJ, Jänig W, Michaelis M (1994) Respiratory modulation in the activity of sympathetic neurones. Prog Neurobiol 43:567-606.

Haselton JR, Guyenet PG (1989) Central respiratory modulation of medullary sympathoexcitatory neurons in rat. Am J Physiol 256:R739-R750.

Jean A (2001) Brain stem control of swallowing: neuronal network and cellular mechanisms. Physiol Rev 81:929-969.

Jean A, Dallaporta M (2013) Brainstem control of deglutition: swallowing pattern generator. In: Principles of deglutition: a multidisciplinary text for swallowing and its disorders (Shaker R, Belafsky PC, Postma GN, Easterling C, eds), pp 67-87. New York: Springer.

Jiang C, Lipski J (1990) Extensive monosynaptic inhibition of ventral respiratory group neurons by augmenting neurons in the Bötzinger complex in the cat. Exp Brain Res 81:639-648.

Kessler JP, Jean A (1985) Identification of the medullary swallowing regions in the rat. Exp Brain Res 57:256-263.

Kumar NN, Bowman BR, Goodchild AK (2012) Combined in situ hybridization and immunohistochemistry in rat brain tissue using digoxigeninlabeled riboprobes. In: Visualization techniques: from immunohistochemistry to magnetic resonance imaging (Badoer E, ed), pp 31-52. Totowa, NJ: Humana.

Lipski J, Kanjhan R, Kruszewska B, Rong W (1996) Properties of presympathetic neurones in the rostral ventrolateral medulla in the rat: an intracellular study 'in vivo.' J Physiol 490:729-744.

Menuet C, Le S, Dempsey B, Connelly AA, Kamar JL, Jancovski N, Bassi JK, Walters K, Simms AE, Hammond A, Fong AY, Goodchild AK, McMullan S, Allen AM (2017) Excessive respiratory modulation of blood pressure triggers hypertension. Cell Metab 25:1-10.

Miyawaki T, Pilowsky P, Sun QJ, Minson J, Suzuki S, Arnolda L, LlewellynSmith I, Chalmers J (1995) Central inspiration increases barosensitivity of neurons in rat rostral ventrolateral medulla. Am J Physiol 268:R909-R918.

Miyawaki T, Goodchild AK, Pilowsky PM (2002) Evidence for a tonic GABA-ergic inhibition of excitatory respiratory-related afferents to presympathetic neurons in the rostral ventrolateral medulla. Brain Res 924:56-62.

Moraes DJ, da Silva MP, Bonagamba LG, Mecawi AS, Zoccal DB, AntunesRodrigues J, Varanda WA, Machado BH (2013) Electrophysiological properties of rostral ventrolateral medulla presympathetic neurons modulated by the respiratory network in rats. J Neurosci 33:19223-19237.

Oku Y, Tanaka I, Ezure K (1994) Activity of bulbar respiratory neurons during fictive coughing and swallowing in the decerebrate cat. J Physiol 480:309-324.

Ono K, Shiba K, Nakazawa K, Shimoyama I (2006) Synaptic origin of the respiratory-modulated activity of laryngeal motoneurons. Neuroscience 140:1079-1088.

Ono T, Ishiwata Y, Inaba N, Kuroda T, Nakamura Y (1998) Modulation of the inspiratory-related activity of hypoglossal premotor neurons during ingestion and rejection in the decerebrate cat. J Neurophysiol 80:48-58.

Papp EA, Leergaard TB, Calabrese E, Johnson GA, Bjaalie JG (2014) Waxholm space atlas of the Sprague Dawley rat brain. Neuroimage 97:374-386.

Richter DW (1982) Generation and maintenance of the respiratory rhythm. J Exp Biol 100:93-107.

Rybak IA, Abdala AP, Markin SN, Paton JF, Smith JC (2007) Spatial organization and state-dependent mechanisms for respiratory rhythm and pattern generation. Prog Brain Res 165:201-220.

Saha M, Menuet C, Sun QJ, Burke PG, Hildreth CM, Allen AM, Phillips JK (2019) Respiratory sympathetic modulation is augmented in chronic kidney disease. Respir Physiol Neurobiol 262:57-66.

Saito Y, Ezure K, Tanaka I, Osawa M (2003) Activity of neurons in ventrolateral respiratory groups during swallowing in decerebrate rats. Brain Dev 25:338-345.

Simms AE, Paton JF, Pickering AE, Allen AM (2009) Amplified respiratorysympathetic coupling in the spontaneously hypertensive rat: does it contribute to hypertension? J Physiol 587:597-610.

Simms AE, Paton JF, Allen AM, Pickering AE (2010) Is augmented central respiratory-sympathetic coupling involved in the generation of hypertension? Respir Physiol Neurobiol 174:89-97.

Smith JC, Abdala AP, Borgmann A, Rybak IA, Paton JF (2013) Brainstem respiratory networks: building blocks and microcircuits. Trends Neurosci $36: 152-162$

Stanek E 4th, Cheng S, Takatoh J, Han BX, Wang F (2014) Monosynaptic premotor circuit tracing reveals neural substrates for oro-motor coordination. eLife 3:e02511.

Sugiyama Y, Shiba K, Nakazawa K, Suzuki T, Umezaki T, Ezure K, Abo N, Yoshihara T, Hisa Y (2011) Axonal projections of medullary swallowing neurons in guinea pigs. J Comp Neurol 519:2193-2211.

Sun QJ, Berkowitz RG, Pilowsky PM (2008) GABAA mediated inhibition and post-inspiratory pattern of laryngeal constrictor motoneurons in rat. Respir Physiol Neurobiol 162:41-47.

Sun QJ, Bautista TG, Berkowitz RG, Zhao WJ, Pilowsky PM (2011) The temporal relationship between non-respiratory burst activity of expiratory laryngeal motoneurons and phrenic apnoea during stimulation of the superior laryngeal nerve in rat. J Physiol 589:1819-1830.

Tago H, McGeer PL, McGeer EG, Akiyama H, Hersh LB (1989) Distribution of choline acetyltransferase immunopositive structures in the rat brainstem. Brain Res 495:271-297.

Umezaki T, Matsuse T, Shin T (1998) Medullary swallowing-related neurons in the anesthetized cat. Neuroreport 9:1793-1798.

Wu J, Capelli P, Bouvier J, Goulding M, Arber S, Fortin G (2017) A V0 core neuronal circuit for inspiration. Nat Commun 8:544.

Yoshihara M, Yoshihara M (2018) 'Necessary and sufficient' in biology is not necessarily necessary: confusions and erroneous conclusions resulting from misapplied logic in the field of biology, especially neuroscience. J Neurogenet 32:53-64.

Zoccal DB, Paton JF, Machado BH (2009) Do changes in the coupling between respiratory and sympathetic activities contribute to neurogenic hypertension? Clin Exp Pharmacol Physiol 36:1188-1196. 